\title{
Funktionelle Charakterisierung von Patienten mit isoliert postkapillärer oder kombiniert post- und präkapillärer pulmonaler Hypertonie
} Functional characterization of patients with isolated post-capillary or combined post-capillary and pre-capillary pulmonary hypertension

\section{(두) (i) $(:)$}

Autoren

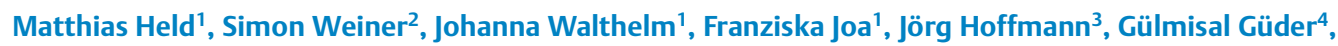
Elena Pfeuffer-Jovic ${ }^{1}$

Institute

1 Medizinische Klinik mit Schwerpunkt Pneumologie und Beatmungsmedizin, Standort Missioklinik, Klinikum Würzburg Mitte gGmbH

2 Institut für Diagnostische und Interventionelle Neuroradiologie, Universitätsklinikum Würzburg

3 Klinik für Thorax-, Herz- und Thorakale Gefäßchirurgie, Universitätsklinikum Würzburg

4 Medizinische Klinik und Poliklinik I, Schwerpunkt Kardiologie, Universitätsklinikum Würzburg

Schlüsselwörter

Pulmonale Hypertonie, Linksherzerkrankung, HFpEF, PH-LHD

Key words

pulmonary hypertension, left heart disease, HFpEF, PH-LHD

Bibliografie

Dtsch Med Wochenschr 2021; 146: e88-e94

DOI 10.1055/a-1555-0345

ISSN 0012-0472

(C) 2021. The Author(s).

This is an open access article published by Thieme under the terms of the Creative Commons Attribution-NonDerivative-NonCommercial License, permitting copying and reproduction so long as the original work is given appropriate credit. Contents may not be used for commecial purposes, or adapted, remixed, transformed or built upon. (https://creativecommons.org/licenses/by-nc-nd/4.0/)

Georg Thieme Verlag KG, Rüdigerstraße 14,

70469 Stuttgart, Germany

Korrespondenzadresse

PD Dr. Matthias Held

Medizinische Klinik mit Schwerpunkt Pneumologie und Beatmungsmedizin, Standort Missioklinik, Klinikum Würzburg Mitte gGmbH, Salvatorstraße 7, 97074 Würzburg, Deutschland

Tel.: +49/931/7912811

matthias.held@kwm-klinikum.de

Zusätzliches Material finden Sie unter https://doi.org/10.1055/a-1555-0345

\section{ZUSAMMENFASSUNG}

Hintergrund Nach Empfehlungen der letzten PH-Weltkonferenz wird die Differenzierung von isoliert postkapillärer (Ipc) und kombiniert post- und präkapillärer ( $\mathrm{Cpc}$ ) PH allein nach pulmonal-vaskulärem Widerstand (PVR) empfohlen. Ziel dieser Arbeit war es, Patienten mit IpcPH und $\mathrm{CpcPH}$ nach den aktualisierten Empfehlungen basierend auf der letzten Weltkonferenz für pulmonale Hypertonie ( $\mathrm{PH}$ ) hämodynamisch und im Rahmen einer explorativen Datenanalyse funktionell zu charakterisieren.

Methoden Evaluiert wurden Patienten, die sich von 2008 bis 2015 in der PH-Ambulanz der Missioklinik Würzburg mit Verdacht auf PH zur leitlinienkonformen Diagnostik vorstellten. Analysiert wurden alle Patienten mit einem pulmonal-arteriellen Mitteldruck (mPAP) von $\geq 25 \mathrm{mmHg}$ und einem pulmonalkapillären Verschlussdruck (PCWP) $>15 \mathrm{mmHg}$. Es erfolgte der Vergleich anthropometrischer, hämodynamischer und funktioneller Daten aus 6-Minuten-Gehtest (6MWT), Spiroergometrie und Echokardiografie bei IpcPH und $\mathrm{CpcPH}$.

Ergebnisse Von 726 Patienten zeigten 58 eine postkapilläre PH: IpcPH: $n=20 ; C p c P H: ~ n=38$. Patienten mit IpcPH besaßen einen signifikant niedrigeren mPAP und PVR als Patienten mit $\mathrm{CpcPH}$. Der Herzindex war in der $\mathrm{CpcPH}$-Gruppe niedriger als in der IpcPH-Gruppe. Die funktionelle Kapazität beider Gruppen war nicht verschieden. Patienten mit $\mathrm{CpcPH}$ hatten einen größeren rechts-linksatrialen Flächen(RA/LA)-Quotienten.

Diskussion und Schlussfolgerung Trotz höherem mPAP und PVR von Patienten mit $\mathrm{CpcPH}$ findet sich keine schlechtere funktionelle Kapazität als bei IpcPH. Bei Patienten mit Linksherzerkrankungen und $\mathrm{PH}$ kann ein erhöhter RA/LA-Index ein möglicher Hinweis für eine $\mathrm{CpcPH}$ sein und Anlass zur invasiven Diagnostik geben.

\section{ABSTRACT}

Background The World Conference on $\mathrm{PH}$ recommended differentiation of isolated postcapillary (IPC) and combined post- and precapillary (Cpc) $\mathrm{PH}$ according to pulmonary vascular resistance alone. The aim of this study was the haemodynamic and functional characterization of patients diagnosed 
IpcPH and $\mathrm{CpcPH}$ according to the current recommendation of the latest World Symposium on Pulmonary Hypertension (PH) with an exploratory data analysis.

Methods We evaluated all consecutive patients presenting at the $\mathrm{PH}$ outpatient clinic of Mission Medical Hospital from 2008-2015. All received a complete diagnostic work-up according to the guidelines. We analyzed data of patients with $\mathrm{mPAP} \geq 25 \mathrm{mmHg}$ and pulmonary capillary wedge pressure $(P C W P)>15 \mathrm{mmHg}$. We compared anthropometric, hemodynamic and functional data of six-minute walking test (6 MWT), cardiopulmonary exercise testing (CPET) and echocardiography of patients with IpcPH and $\mathrm{CpcPH}$.
Results Out of 726 patients 58 showed a postcapillary $\mathrm{PH}$ : IpcPH: $n=20$; $C p c P H: n=38$. Patients with IpcPH had a significantly lower mPAP and PVR than patients with $\mathrm{CPcPH}$. Cardiac index was lower in the $\mathrm{Cpc}-\mathrm{PH}$ group compared to the IpcPH group. Functional capacity did not differ. $\mathrm{CpcPH}$ patients showed a higher right/left atrial area (RA/LA)-ratio.

Discussion and conclusion Although $\mathrm{CPcPH}$ patients showed higher values of mPAP and PVR functional capacity was not worse than in patients with IPcPH. In patients with $\mathrm{PH}$ due to left heart disease an elevated RA/LA ratio may indicate $\mathrm{CpcPH}$ and invasive diagnostic work-up should be considered.

\section{Einleitung}

Gemäß der letzten Weltkonferenz für pulmonale Hypertonie (PH) wird die postkapilläre $\mathrm{PH}(\mathrm{pcPH})$ allein nach dem pulmonal-vaskulären Widerstand (PVR) in eine von der linken Herzkammer passiv fortgeleitete Druckerhöhung und eine sekundär durch pulmonalvaskuläres Remodeling induzierte Widerstandserhöhung unterteilt [1]. Sie folgt pathophysiologischen Überlegungen und reflektiert damit einhergehende Beobachtungen unterschiedlicher Prognose [2] und möglicherweise anzunehmender therapeutischer Konsequenzen [3, 4].

Nach der bisher gültigen Leitlinie lag das Definitionskriterium der PH bislang bei einem pulmonal-arteriellen Mitteldruck (mPAP) $\geq 25 \mathrm{mmHg}$ [5]. Bei Patienten mit einem zudem vorliegenden pulmonal-kapillärer Verschlussdruck (PCWP) von $>15 \mathrm{mmHg}$ und damit bestehender pcPH unterscheidet die aktualisierte Empfehlung gemäß dem „ $6^{\text {th }}$ World Symposium on Pulmonary Hypertension“ hämodynamisch eine isoliert postkapilläre (IPC) PH mit einem PVR $<3$ Wood-Einheiten (WU) von einer kombiniert postund präkapillären (Cpc) PH mit einem PVR $\geq 3$ WU [1].

Bei pcPH besteht die Empfehlung zur Therapie der zugrunde liegenden Linksherzerkrankung [1, 5]. Während bei Valvulopathien die Klappensanierung [6] und bei systolischer Linksherzinsuffizienz eine medikamentöse Therapie empfohlen ist [7], existiert für die diastolische Linksherzinsuffizienz (HFpEF) außer einer diuretikabasierten Therapie keine spezifische Behandlungsoption [7].

Gerade bei der HFpEF stellt sich die Frage, ob eine gezielte Therapie einer $\mathrm{pcPH}$ vorteilhaft sein könnte. Zahlreiche Studien konnten bei pcPH keinen Vorteil einer PAH-spezifischen Therapie mit Medikation erkennen lassen [8-10].

Daten einer italienischen Studie [11], Registerdaten [3, 12] und pathophysiologisch basierte Überlegungen $[4,13]$ lassen jedoch davon ausgehen, dass eine medikamentöse spezifische Therapie einer $\mathrm{CpcPH}$ mit Erhöhung des PVR erfolgversprechend sein könnte. Dazu laufen bereits weitere Studien [1, 14].

Daten zu Häufigkeit und funktioneller Charakterisierung einer IpcPH und $\mathrm{CpcPH}$ nach der aktuellen Definition sind rar. Die aktuelle Untersuchung sollte das Vorkommen von IpcPH und $\mathrm{CpcPH}$ nach der neuen Definition im Kollektiv einer $\mathrm{PH}$-Ambulanz analy- sieren und die Gruppen im Rahmen einer explorativen Datenanalyse charakterisieren.

\section{Patienten und Methodik}

Es wurden die Daten aller erwachsenen Patienten erfasst und retrospektiv ausgewertet, die sich mit Verdacht auf $\mathrm{PH}$ in der $\mathrm{PH}$ Ambulanz der Missioklinik Würzburg zwischen dem 01.01.2008 und 31.07.2015 vorgestellt hatten. Alle Patienten hatten schriftlich ihr Einverständnis zur Datenanalyse erteilt. Die Untersuchung steht im Einklang mit der Deklaration von Helsinki gemäß der damals gültigen Version (2008), und ein Ethikvotum (Nr. 20191217011) wurde am 03.12.2019 eingeholt.

\section{Diagnostik}

Bei den insgesamt 726 Patienten wurden im Rahmen der PHAbklärung eine Echokardiografie [15], ein 6 MWT [16], eine Spiroergometrie [17] und ein Rechtsherzkatheter [18] gemäß Leitlinienempfehlung durchgeführt [5, 19]. Mittels Echokardiografie wurden nicht nur Standardwerte wie die Ejektionsfraktion (EF), diastolische Dysfunktion und die rechtsventrikuläre Funktion mittels tricuspid annular plane systolic excursion (TAPSE) erhoben, sondern auch endsystolisch die links- (LA) und rechtsatriale Fläche (RA) im 4-Kammerblick erhoben, um einen RA/LA-Quotienten zu bilden.

Die Diagnose einer PH erfolgte gemäß der letzten Guideline bei einem mPAP $\geq 25 \mathrm{mmHg}$ [5], wohingegen bei zudem vorliegendem PCWP von $>15 \mathrm{mmHg}$ die Klassifikation in IpcPH und $\mathrm{CpcPH}$ nach den aktuellen Empfehlungen der PH-Weltkonferenz gemäß PVR durchgeführt wurde [1]. Eine mögliche thromboembolische Vaskulopathie wurde mittels Ventilations-Perfusionsszintigrafie bei allen Patienten gemäß diagnostischem Work-up bei erhöhtem mPAP ausgeschlossen.

Die Patienten ohne Nachweis einer $\mathrm{PH}$, allerdings mit zugrunde liegender Linksherzerkrankung dienten als Kontrollkollektiv zum Vergleich mit den Patienten mit pcPH. Die Häufigkeiten der Komorbiditäten in den jeweiligen Kollektiven wurden ermittelt. Zudem erfolgte der Vergleich anthropometrischer, hämodynamischer und funktioneller Daten der Patienten mit IpcPH und $\mathrm{CpcPH}$. 


\section{Statistische Auswertung}

Die statistische Auswertung erfolgte mithilfe von SPSS Statistics 23 und SPSS Statistics 26 (IBM, USA). Zur grafischen Darstellung wurde Microsoft Excel 2013 (Microsoft Corporation, USA) verwendet.

Jeder Parameter wurde einzeln durch QQ-Plot und Kolmogorow-Smirnow-Test auf Normalverteilung hin überprüft. Zur Darstellung der Daten, für die eine Normalverteilung angenommen werden kann, sind jeweils Mittelwert (MW) und Standardabweichung (SD) angegeben.

Beim Vergleich unabhängiger Stichproben wurde ein t-Test verwendet, neben der Mittelwertdifferenz wurde jeweils das entsprechende $95 \%$-Konfidenzintervall angegeben. Bei fehlenden Werten erfolgte ein paarweiser Fallausschluss. Zur Abschätzung der Stärke des Zusammenhangs zweier kategorialer Parameter wurde die Odds Ratio mit korrespondierendem $95 \%$-Konfidenzintervall verwendet. Als Signifikanzniveau wurde $\alpha=0,05$ gewählt. Im Rahmen der explorativen Analyse erfolgte keine Adjustierung der $\mathrm{p}$-Werte für multiples Testen.

\section{Ergebnisse}

Von den im Untersuchungszeitraum 2008-2015 vorgestellten 726 Patienten wurde bei 556 Patienten eine $\mathrm{PH}$ mit $m P A P \geq 25 \mathrm{mmHg}$ invasiv bestätigt, bei 58 Patienten lag eine pcPH vor. Das durchschnittliche Alter dieser Gruppe betrug 70,9 $\pm 10,2$ Jahre. 72,4\% der Patienten waren weiblich. 69,0\% der Patienten waren in der WHO-Funktionsklasse III. In der Mehrzahl der Fälle (55,2\%) lag eine HFpEF vor, nur in 13,8\% fand sich eine systolische Herzinsuffizienz. Das häufigste linkskardiale Klappenvitium war eine Mitralklappeninsuffizienz (37,9\%), gefolgt von Aortenklappeninsuffizienz (12,1\%). Eine vollständige Auflistung der Komorbiditäten und der anthropometrischen Daten der 58 Patienten mit pcPH ist der $>$ Tab. $\mathbf{S} 1$ im Supplement zu entnehmen.

Der Vergleich der pcPH-Kohorte mit den 63 Patienten mit Linksherzerkrankung, aber ausgeschlossener $\mathrm{PH}$ ergab, dass sich neben anthropometrischen Daten auch diastolische und systolische linksventrikuläre Funktionswerte nicht unterschieden, jedoch Patienten mit pcPH größere sowohl rechts- als auch linksseitige Vorhöfe, eine signifikant reduzierte TAPSE und schlechtere funktionelle Kapazität gemäß 6 MWT aufwiesen $(p<0,01)$ $(\triangleright$ Tab. 1).

Unter den 58 Patienten mit PH infolge einer Linksherzerkrankung erfolgte gemäß PVR die Zuordnung von 20 Patienten ins IpcPH-Kollektiv, wohingegen 38 Patienten in die Gruppe der $\mathrm{CpcPH}$ eingeordnet wurden. Beim Vergleich von $\mathrm{CpcPH}$ - und IpcPH-Kollektiven ( $\triangleright$ Tab. 2 ) ergaben sich keine relevanten Unterschiede bezüglich anthropometrischer oder funktioneller Daten. Allerdings lag im CpcPH-Kollektiv ein höherer mPAP (СрсPH: $41,2 \pm 8,0 \mathrm{mmHg}$, IpcPH: $34,0 \pm 9,5 \mathrm{mmHg})$ und PVR (СрсPH: $5,0 \pm 1,7 \mathrm{WU}$, IpcPH: 1,7 $\pm 0,9 \mathrm{WU})$ vor $(\mathrm{p}<0,001)$. In Einklang damit fand sich bei Patienten mit $\mathrm{CpcPH}$ ein größerer RA/LAQuotient (IpcPH: 0,83 $\pm 0,34, \mathrm{CpcPH}: 1,15 \pm 0,47, \mathrm{p}=0,02)$. Der Herzindex $(\mathrm{HI})$ war bei $\mathrm{CpcPH}$ niedriger als bei IpcPH $(\mathrm{p}=0,01)$.

Prozentual gesehen zählte die HFpEF sowohl im CpcPH-Kollektiv als auch im IpcPH-Kollektiv mit jeweils 50,0\% bzw. 65,0\% zu den häufigsten linkskardialen Pathologien und führenden Ursachen der PH ( $\triangleright$ Tab. 3). Linkskardiale Klappenvitien waren lediglich mit 25,0\% bei Ipc sowie 36,8\% bei Cpc ursächlich für die $\mathrm{PH}$.

Im Hinblick auf andere relevante Komorbiditäten zeigte sich in der Gruppe der IpcPH unter anderem eine hohe Prävalenz von Adipositas (BMI $>30 \mathrm{~kg} / \mathrm{m}^{2}$ ) $(65,0 \%)$, Schlafapnoesyndrom (40,0\%), Niereninsuffizienz (40,0\%) und KHK (30,0\%) mit dementsprechender Odds Ratio. Im Kollektiv der CpcPH traten gehäuft arterielle Hypertonie (76,3\%), Vorhofflimmern (31,6\%), Schilddrüsenpathologien (44,7\%) und COPD (13,2\%) auf.

Auch bei der getrennten Betrachtung der Patienten mit pcPH und HFpEF (Online Supplement - Tab.S 2) fanden sich lediglich signifikante hämodynamische Unterschiede beim Vergleich von $\mathrm{CpcPH}$ und IpcPH. So besaßen Patienten mit CpcPH und HFpEF einen höheren mPAP (CрсPH: 42,3 $\pm 7,3 \mathrm{mmHg}$; IрсPH: $33,5 \pm 9,4 \mathrm{mmHg})$, PVR (СрсPH: 4,8 $\pm 1,6 \mathrm{WU}$; ІрсPH: $1,7 \pm 0,9 \mathrm{WU})$ und diastolischen Druckgradienten (DPG) (CpcPH: $7,7 \pm 6,8 \mathrm{mmHg}$; IpcPH: $-0,3 \pm 6,5 \mathrm{mmHg}$ ) als Patienten mit IpcPH und zugrunde liegender HFpEF ( $p<0,01)$. Bei Patienten mit pcPH infolge eines linksseitigen Herzklappenvitiums (Online Supplement $\mathbf{T a b} . \mathbf{S} 3$ ) fanden sich bei $\mathrm{CpcPH}$ neben einem höheren PVR (CpcPH: 4,8 \pm 1,9 WU; IpcPH: 1,7 $\pm 1,2 \mathrm{WU}$ ) ein niedrigerer Herzindex (CpcPH: 2,4 $\pm 0,41 \mathrm{~min}^{-1} \mathrm{~m}^{-2}$; IpcPH: 3,3 \pm $0,31 \mathrm{~min}^{-1} \mathrm{~m}^{-2}$ ) und ein höherer RA/LA-Quotient (CpcPH: 1,15 \pm 0,5; IpcPH: 0,54 $\pm 0,2)$ im Vergleich zu Patienten mit IpcPH und Klappenvitium $(p<0,05)$.

Die funktionelle Kapazität war sowohl bei IpcPH als auch $\mathrm{CpcPH}$ bei HFpEF und auch linkskardialem Klappenvitium nicht unterschiedlich.

\section{Diskussion}

Die pcPH stellt global die häufigste Form der PH dar [20]. Spezifische Therapieansätze, die über die Therapie der Grunderkrankung $[1,5]$ hinausgehen, fehlen. Daher stellt die pcPH eine Gruppe von besonderem wissenschaftlichem Interesse dar.

Über 72 \% der Patienten mit pcPH befinden sich in einer höheren WHO-Funktionsklasse, was die Notwendigkeit weiterer wissenschaftlicher Aktivität in Bezug auf dieses Krankheitsbild betont. Obwohl die pcPH weltweit die häufigste Lungenhochdruckgruppe ist, sind lediglich 58 Patienten aus dem Kollektiv von 556 bestätigen Patienten mit PH einer pcPH zuzuordnen. Die gewonnenen Daten stammen aus einem Kollektiv, das mit bereits bestehendem Verdacht auf eine PH in ein spezialisiertes Zentrum eingewiesen wurde. Eine genaue Prävalenz der pcPH und im speziellen $\mathrm{CpcPH}$ und IpcPH bei Linksherzerkrankungen kann daraus nicht hervorgehen. Der eher geringere Anteil der pcPH in dem untersuchten Kollektiv erklärt sich unter anderem durch einen Selektionsbias. Aufgrund bis dato nicht zugelassener gezielter $\mathrm{PH}$-Medikamente für dieses $\mathrm{PH}$-Kollektiv werden in einer Spezialambulanz für $\mathrm{PH}$ solche Patienten in der Regel vor allem dann vorgestellt, wenn die $\mathrm{PH}$ besonders schwer erscheint oder aber eine andere Form der PH vermutet wird. Patienten, bei denen eine $\mathrm{pcPH}$ wahrscheinlich erscheint, werden aufgrund einer klaren Empfehlung zur Therapie der Grunderkrankung [1, 5] nicht primär einer PH-Ambulanz zugewiesen. 
- Tab. 1 Vergleich zwischen pcPH-Patienten sowie der Kontrollgruppe mit Linksherzerkrankung ohne PH (LHDoPH).

\begin{tabular}{|c|c|c|c|c|c|c|c|}
\hline & \multicolumn{2}{|c|}{ pcPH } & \multicolumn{2}{|c|}{ LHDoPH } & \multicolumn{3}{|c|}{$\begin{array}{l}\text { pcPH vs. LHDoPH } \\
\text { t-Test bzw. Odds Ratio* }\end{array}$} \\
\hline & $\mathbf{n}$ & $\begin{array}{l}\mathrm{MW} \pm \mathrm{SD} \\
\text { bzw. } \times(x / n)^{*}\end{array}$ & $\mathbf{n}$ & $\begin{array}{l}\mathrm{MW} \pm \mathrm{SD} \\
\text { bzw. } \times(x / n)^{*}\end{array}$ & $\begin{array}{l}\text { MW-Differenz } \\
\text { bzw. OR }\end{array}$ & $\begin{array}{l}95 \%-K I \text { der } \\
\text { MW-Differenz } \\
\text { bzw. der OR* }\end{array}$ & p-Wert \\
\hline Alter [Jahre] & 58 & $70,9 \pm 10,2$ & 63 & $69,3 \pm 10,7$ & 1,6 & {$[-2,2 ; 5,4]$} & $p=0,40$ \\
\hline Weiblich* & 58 & $42(72,4 \%)^{*}$ & 63 & $42(66,7 \%)^{*}$ & $1,3^{*}$ & {$[0,6 ; 2,9]^{*}$} & $-*$ \\
\hline 6-Minuten-Gehtest [m] & 57 & $309,7 \pm 116,5$ & 57 & $370,2 \pm 113,2$ & $-60,4$ & {$[-103,1 ;-17,8]$} & $p=0,006$ \\
\hline $\mathrm{RA}\left[\mathrm{cm}^{2}\right]$ & 51 & $24,1 \pm 7,8$ & 59 & $17,3 \pm 7,3$ & 6,8 & {$[3,9 ; 9,6]$} & $p<0,001$ \\
\hline $\mathrm{LA}\left[\mathrm{cm}^{2}\right]$ & 50 & $25,5 \pm 11,0$ & 57 & $19,7 \pm 8,0$ & 5,8 & {$[2,1 ; 9,5]$} & $p=0,002$ \\
\hline RA/LA & 49 & $1,05 \pm 0,46$ & 57 & $0,90 \pm 0,35$ & 0,14 & {$[-0,02 ; 0,30]$} & $p=0,08$ \\
\hline $\mathrm{EF}[\%]$ & 51 & $55,5 \pm 12,1$ & 59 & $58,0 \pm 11,0$ & $-2,7$ & {$[-7,2 ; 1,7]$} & $p=0,23$ \\
\hline$E / E^{\prime}$ & 52 & $12,1 \pm 6,9$ & 55 & $10,3 \pm 3,8$ & 1,8 & {$[-0,3 ; 4,0]$} & $p=0,10$ \\
\hline TAPSE [mm] & 54 & $19,2 \pm 5,7$ & 61 & $24,0 \pm 5,2$ & $-4,8$ & {$[-6,8 ;-2,7]$} & $\mathrm{p}<0,001$ \\
\hline
\end{tabular}

"Für den nominalskalierten Parameter Geschlecht ist die absolute Anzahl × sowie in Klammern der relative Anteil x/n gegeben. Als Vergleichsparameter sind die Odds Ratio (OR) sowie das $95 \%$-Konfidenzintervall der Odds Ratio angegeben.

Die nach der PH-Weltkonferenz von 2018 aktualisierte Definition der pcPH [1] unterteilt IpcPH und $\mathrm{CpcPH}$ nun lediglich nach der Höhe des PVR. Dies ist als Weiterentwicklung der wissenschaftlichen Auseinandersetzung bisheriger negativer Studiendaten aus der Untersuchung gezielter PH-Therapeutika zu verstehen. Es konnte gezeigt werden, dass Patienten mit HFpEF ohne Vorliegen einer $\mathrm{PH}[10,11]$, und auch Patienten mit moderater $\mathrm{PH}$ ohne Rechtsherzdysfunktion [9] nicht von einer gezielten $\mathrm{PH}$ Therapie profitieren. Das Kollektiv der letztgenannten Arbeit würde man als Kollektiv mit IpcPH verstehen. Aus dem Verständnis dieser Daten wuchs die Überzeugung, dass ein Einsatz von für die PAH zugelassenen und direkt an den Lungengefäßen via Vasorelaxation angreifenden Medikamenten bei einer $\mathrm{PH}$ infolge einer Linksherzerkrankung nur bei einem relevant erhöhten PVR sinnvoll erscheint.

Die neue Definition der letzten $\mathrm{PH}-$ Weltkonferenz trennt die IpcPH und CpcPH allein nach dem PVR [1]. Zudem konnte gezeigt werden, dass Patienten mit CpсPH einen signifikant höheren mPAP haben als Patienten mit IpcPH. Dies passt zum Konzept, dass man heutzutage eine kontinuierliche Entwicklung ausgehend von einer diastolischen linksventrikulären Dysfunktion über eine IpcPH hin zu einer CpcPH annimmt $[4,12]$, bei der aufgrund der längeren Laufzeit der Erkrankung ein höherer mPAP bei $\mathrm{CpcPH}$ Folge der Progression ist.

Man geht heute davon aus, dass es neben einer Suszeptibilität für die Entwicklung einer PH verschiedener Ereignisse bedarf, um ein vaskuläres Remodeling auszulösen [21]. Einer dieser auslösenden Faktoren ist ein dauerhaft erhöhter PCWP, der bei entsprechender Empfänglichkeit ein Remodeling und damit eine Erhöhung des PVR induziert.

Die Patienten mit IpcPH und $\mathrm{CpcPH}$ besitzen ähnliche Ausgangsvoraussetzungen, erkennbar an nicht unterschiedlichen anthropometrischen Daten. Trotz signifikanter Unterschiede des PVR und mPAP zeigte sich die funktionelle Kapazität bei IpcPH und $\mathrm{CpcPH}$ ähnlich. Diese Beobachtung ergibt sich auch bei der getrennten Betrachtung von IpcPH/CpcPH bei HFpEF einerseits und Klappenvitien andererseits (Online Supplement $>$ Tab. $\mathbf{S} 2$ und $>$ Tab. S 3) andererseits.

Obwohl die funktionelle Kapazität bei $\mathrm{CpcPH}$ und IpcPH ähnlich ist, ist eine Unterscheidung zwischen den Gruppen im klinischen Alltag essenziell, da bereits gezeigt werden konnte, dass mit zunehmendem PVR bei HFpEF und PH mit einer Verschlechterung der Prognose zu rechnen ist [2]. Die schlechteste Prognose besitzen Patienten mit zusätzlicher rechtsventrikulärer Dysfunktion [22].

Trotz Sichtung über einen größeren Zeitraum ist die Zahl von Patienten mit CpcPH überschaubar. Dies macht verständlich, dass die Rekrutierung in klinische Studien zur pcPH anspruchsvoll ist und eine längere Rekrutierungsdauer benötigt.

Eine neben der LA-Vergößerung vorliegende zusätzliche RAVergrößerung ist unter anderem der pulmonalvaskulären Druckbelastung geschuldet. Möglicherweise kann der RA/LA-Quotient Hinweise auf eine $\mathrm{CpcPH}$ geben, da diese einen erhöhten RA/LAQuotienten haben. Somit kann ein erhöhter RA/LA-Quotient auch bei Patienten mit Linksherzerkrankungen in der kardiologischen Praxis den Anstoß geben, eine weitere invasive Abklärung zu veranlassen.

\section{Limitationen}

Die monozentrische, retrospektive Analyse sowie die relativ eingeschränkten Fallzahlen stellen gewisse Limitationen dar. Dennoch sind die Daten robust, da alle Patienten ein komplettes diagnostisches Work-up gemäß der aktuellen Leitlinie erhielten. Eine weitere Studie mit größerem pcPH-Kollektiv zur genaueren Charakterisierung ist allerdings bereits geplant. 
- Tab. 2 Vergleich der Patienten mit IpcPH und CpcPH.

\begin{tabular}{|c|c|c|c|c|c|c|c|}
\hline & \multicolumn{2}{|c|}{ IpcPH } & \multicolumn{2}{|c|}{ СрсPH } & \multicolumn{3}{|c|}{$\begin{array}{l}\text { IpcPH vs. CpcPH } \\
\text { t-Test bzw. Odds Ratio* }\end{array}$} \\
\hline & n & $\begin{array}{l}\mathrm{MW} \pm \mathrm{SD} \\
\mathrm{bzw} . \times(\mathrm{x} / \mathrm{n})^{*}\end{array}$ & $\mathbf{n}$ & $\begin{array}{l}M W \pm S D \\
\text { bzw. } \times(x / n)^{*}\end{array}$ & $\begin{array}{l}\text { MW-Differenz } \\
\text { bzW. OR* }\end{array}$ & $\begin{array}{l}95 \%-K I \text { der } \\
\text { MW-Differenz } \\
\text { bzW. der OR }{ }^{*}\end{array}$ & p-Wert \\
\hline \multicolumn{8}{|l|}{ Anthropometrie } \\
\hline Alter [a] & \multirow[t]{3}{*}{20} & $67,6 \pm 13,8$ & \multirow[t]{3}{*}{38} & $72,6 \pm 7,3$ & $-5,1$ & {$[-11,9 ; 1,7]$} & $p=0,14$ \\
\hline Weiblich* & & $13(65,0 \%)^{*}$ & & $29(76,3 \%)^{*}$ & $1,7^{*}$ & {$[0,5 ; 5,7]^{*}$} & $-*$ \\
\hline BMI $\left[\mathrm{kg} / \mathrm{m}^{2}\right]$ & & $33,3 \pm 9,0$ & & $30,3 \pm 5,1$ & 3,0 & {$[-1,5 ; 7,4]$} & $p=0,18$ \\
\hline \multicolumn{8}{|l|}{ WHO-Funktionsklasse } \\
\hline I* $^{*}$ & \multirow[t]{4}{*}{20} & $0^{*}$ & \multirow[t]{4}{*}{38} & $0^{*}$ & $-{ }^{*}$ & $-{ }^{*}$ & $-{ }^{*}$ \\
\hline $\mathrm{II}^{*}$ & & $4(20,0 \%)^{*}$ & & $12(31,6 \%)^{*}$ & $1,8^{*}$ & {$[0,5 ; 6,7]^{*}$} & $-{ }^{*}$ \\
\hline $\mathrm{III}^{*}$ & & $15(75,0 \%)^{*}$ & & $25(65,8 \%)^{*}$ & $0,6^{*}$ & {$[0,2 ; 2,2]^{*}$} & $-{ }^{*}$ \\
\hline IV* & & $1(5,0 \%)^{*}$ & & $1(2,6 \%)^{*}$ & $0,5^{*}$ & {$[0,0 ; 8,7]^{*}$} & $-*$ \\
\hline \multicolumn{8}{|l|}{ Hämodynamik } \\
\hline mPAP [mmHg] & \multirow[t]{5}{*}{20} & $34,0 \pm 9,5$ & \multirow[t]{5}{*}{38} & $41,2 \pm 8,0$ & $-7,9$ & {$[-12,7 ;-3,2]$} & $p=0,001$ \\
\hline PCWP [mmHg] & & $21,8 \pm 4,9$ & & $20,1 \pm 3,3$ & 1,1 & {$[-0,5 ; 3,8]$} & $p=0,13$ \\
\hline DPG [mmHg] & & $0,0 \pm 6,4$ & & $7,1 \pm 5,9$ & $-7,1$ & {$[-10,4 ;-3,7]$} & $\mathrm{p}<0,001$ \\
\hline PVR [WU] & & $1,7 \pm 0,9$ & & $5,0 \pm 1,7$ & $-3,3$ & {$[-4,2 ;-2,4]$} & $p<0,001$ \\
\hline $\mathrm{HI}\left[\mathrm{I} \mathrm{min}^{-1} \mathrm{~m}^{-2}\right]$ & & $2,8 \pm 0,6$ & & $2,3 \pm 0,4$ & 0,5 & {$[0,1 ; 0,8]$} & $p=0,01$ \\
\hline \multicolumn{8}{|l|}{ Funktionelle Parameter } \\
\hline 6-Minutengehstrecke [m] & 20 & $316,6 \pm 112,8$ & 37 & $306,0 \pm 119,8$ & 10,6 & {$[-54,7 ; 75,9]$} & $p=0,75$ \\
\hline $\mathrm{VO}_{2}$ maximal $\left[\mathrm{ml} \mathrm{min}^{-1} \mathrm{~kg}^{-1}\right]$ & 16 & $11,8 \pm 3,2$ & 23 & $12,8 \pm 2,4$ & $-1,0$ & {$[-2,8 ; 0,8]$} & $p=0,27$ \\
\hline \multicolumn{8}{|l|}{ Echokardiografie } \\
\hline RA Fläche $\left[\mathrm{cm}^{2}\right]$ & 17 & $21,4 \pm 6,9$ & 34 & $25,5 \pm 7,9$ & $-4,1$ & {$[-8,6 ; 0,4]$} & $p=0,07$ \\
\hline LA Fläche [cm²] & 16 & $28,8 \pm 16,8$ & 34 & $24,0 \pm 6,7$ & 4,8 & {$[-4,4 ; 14,0]$} & $p=0,29$ \\
\hline RA/LA Quotient & 16 & $0,83 \pm 0,34$ & 33 & $1,15 \pm 0,47$ & $-0,32$ & {$[-0,59 ;-0,05]$} & $p=0,02$ \\
\hline EF [\%] & 17 & $54,5 \pm 13,9$ & 34 & $55,8 \pm 11,3$ & $-1,3$ & {$[-8,6 ; 6,0]$} & $p=0,72$ \\
\hline$E / E^{\prime}$ & 19 & $12,7 \pm 5,1$ & 33 & $11,7 \pm 7,9$ & 1,0 & {$[-3,1 ; 5,0]$} & $p=0,63$ \\
\hline TAPSE [mm] & 19 & $21,0 \pm 5,0$ & 35 & $18,2 \pm 5,9$ & 2,8 & {$[-0,4 ; 6,0]$} & $p=0,09$ \\
\hline \multicolumn{8}{|l|}{ Laborparameter } \\
\hline NT-pro-BNP [pg/ml] & 17 & $2378 \pm 2896$ & 35 & $2756 \pm 2174$ & -378 & {$[-1820 ; 1064]$} & $p=0,60$ \\
\hline
\end{tabular}

* Für die nominalskalierten Parameter Geschlecht und WHO-Klasse sind die Anzahl × sowie in Klammern der Anteil x/n gegeben. Als Vergleichsparameter sind die Odds Ratio (OR) sowie das $95 \%$-Konfidenzintervall der Odds Ratio angegeben.

PcPH stellt eine Erkrankung mit heterogener Ätiologie dar und demnach auch unterschiedlichen Pathomechanismen. In den hier beschriebenen Kollektiven überwiegt allerdings die HFpEF als Ursache der Ipc- bzw. CpcPH, sodass die gewonnenen Erkenntnisse hauptsächlich Patienten mit pcPH und vorliegender diastolischer Dysfunktion repräsentieren. Um die Ergebnisse allerdings auch auf Patienten mit beispielsweise Klappenvitien als Ursache der pcPH zu übertragen, sollten weitere Kollektive mit eben genannter pcPHUrsache betrachtet und verglichen werden.

Definitionskriterium der $\mathrm{PH}$ in dieser Studie war weiterhin ein $\mathrm{mPAP} \geq 25 \mathrm{mmHg}$. Basierend auf zwischenzeitlich publizierten
Daten [23, 24], wurde das Definitionskriterium mittlerweile auf $21 \mathrm{mmHg}$ gesenkt [25]. Laut aktueller Expertenmeinung hat diese Senkung allerdings keine Relevanz für die pcPH [26]. Die Analyse des Gesamtkollektivs von 726 Patienten ergab letztlich nur einen weiteren Patienten mit einem mPAP zwischen 21 und $24 \mathrm{mmHg}$, der gemäß PVR unter das Ipc-Kollektiv fiel (Daten nicht gezeigt).

Die Senkung des mPAP-Schwellenwerts zielt auf eine frühzeitige Diagnosestellung vorwiegend von kollagenoseassoziierter PAH ab, die einem engmaschigeren Monitoring bedarf [25]. Aus einem mPAP zwischen 21 und $24 \mathrm{mmHg}$ resultiert allerdings letztlich 
- Tab. 3 Prävalenz der spezifischen linkskardialen und allgemeinen Komorbiditäten bei Patienten mit Ipc- und CpcPH. Angegeben sind jeweils die absolute und die relative Anzahl sowie die Odds Ratio als Vergleichsparameter.

\begin{tabular}{|c|c|c|c|c|}
\hline & \multirow{2}{*}{$\begin{array}{l}\text { IpcPH }(n=20) \\
x(x / n)\end{array}$} & \multirow{2}{*}{$\begin{array}{l}\text { CpcPH }(n=38) \\
x(x / n)\end{array}$} & \multicolumn{2}{|c|}{ IpcPH vs. СpcPH } \\
\hline & & & Odds Ratio & $\begin{array}{l}95 \%-K I \\
\text { der Odds Ratio }\end{array}$ \\
\hline \multicolumn{5}{|l|}{ Allgemeine Komorbiditäten } \\
\hline Vorhofflimmern & $3(15,0 \%)$ & $12(31,6 \%)$ & 2,6 & {$[0,6 ; 10,7]$} \\
\hline Adipositas & $13(65,0 \%)$ & $19(50,0 \%)$ & 0,5 & {$[0,2 ; 1,6]$} \\
\hline Obesitas-Hypoventilationssyndrom & $2(10,0 \%)$ & $1(2,6 \%)$ & 0,2 & {$[0,0 ; 2,9]$} \\
\hline Schlafapnoe & $8(40,0 \%)$ & $7(18,4 \%)$ & 0,3 & {$[0,1 ; 1,1]$} \\
\hline Arterielle Hypertonie & $14(70,0 \%)$ & $29(76,3 \%)$ & 1,4 & {$[0,4 ; 4,7]$} \\
\hline COPD & $2(10,0 \%)$ & $5(13,2 \%)$ & 1,4 & {$[0,2 ; 7,8]$} \\
\hline Interstitielle Lungenerkrankung & $3(15,0 \%)$ & $1(2,6 \%)$ & 0,2 & {$[0,0 ; 1,6]$} \\
\hline Asthma & $2(10,0 \%)$ & $4(10,5 \%)$ & 1,1 & {$[0,2 ; 6,3]$} \\
\hline KHK & $6(30,0 \%)$ & $8(21,1 \%)$ & 0,6 & {$[0,2 ; 2,1]$} \\
\hline Diabetes mellitus & $5(25,0 \%)$ & $10(26,3 \%)$ & 1,1 & {$[0,3 ; 3,7]$} \\
\hline Niereninsuffizienz (GFR <60 ml/min) & $8(40,0 \%)$ & $11(28,9 \%)$ & 0,6 & {$[0,2 ; 1,9]$} \\
\hline Schilddrüsenerkrankung & $7(35,0 \%)$ & $17(44,7 \%)$ & 1,5 & {$[0,5 ; 4,6]$} \\
\hline \multicolumn{5}{|c|}{ Linkskardiale Komorbiditäten/davon ursächlich für PH } \\
\hline HFrEF & $3(15,0 \%) / 2$ & $5(13,2 \%) / 5$ & 0,9 & {$[0,2 ; 4,0]$} \\
\hline HFpEF & $13(65,0 \%) / 13$ & $19(50,0 \%) / 19$ & 0,5 & {$[0,2 ; 1,6]$} \\
\hline Mitralklappeninsuffizienz & $5(25,0 \%) / 3$ & $17(44,7 \%) / 7$ & 2,4 & {$[0,7 ; 8,0]$} \\
\hline Mitralklappenstenose & $1(5,0 \%) / 1$ & $1(2,6 \%) /-$ & 0,5 & {$[0,0 ; 8,7]$} \\
\hline Aortenklappenstenose & $1(5,0 \%) / 1$ & $5(13,2 \%) / 4$ & 2,9 & {$[0,3 ; 26,5]$} \\
\hline Aortenklappeninsuffizienz & $1(5,0 \%) /-$ & $6(15,8 \%) / 3$ & 3,6 & {$[0,4 ; 31,9]$} \\
\hline
\end{tabular}

Legende: $x=$ absolute Anzahl, $x / n=$ relative Anzahl, OR = Odds Ratio, $95 \%-K I=95 \%$-Konfidenzintervall, HFrEF = heart failure with reduced ejection fraction, $\mathrm{HFpEF}=$ heart failure with preserved ejection fraction.

weder bei pcPH noch bei einer anderen PH-Form eine medikamentöse Therapieempfehlung [26]. Unter diesem Aspekt scheint die Beibehaltung des ursprünglichen mPAP-Definitionskriteriums von $25 \mathrm{mmHg}$ aus praktischen Erwägungen hinreichend.

\section{Fazit für die Praxis}

Trotz bekanntermaßen schlechterer Prognose von Patienten mit $\mathrm{CpcPH}$ gegenüber Patienten mit IpcPH [2], findet sich trotz höheren mPAP und PVR bei CpcPH keine schlechtere funktionelle Kapazität. Bei Patienten mit Linksherzerkrankungen und PH kann ein erhöhter RA/LA-Quotient ein möglicher Hinweis für eine $\mathrm{CpcPH}$ sein und 3Anlass zur weiteren Diagnostik geben.

\section{KERNAUSSAGEN}

- Trotz unterschiedlicher Hämodynamik besitzen Patienten mit CpcPH keine schlechtere funktionelle Kapazität als
Patienten mit IpcPH, gemessen am 6 MWT, WHO-Funktionsstadium sowie anhand spiroergometrischer Daten.

- HFpEF stellt sowohl im CpcPH-Kollektiv als auch im IpcPHKollektiv die häufigste linkskardiale Komorbidität und die führende Ursache der PH dar.

- Patienten mit CpcPH besitzen einen höheren RA/LA-Quotienten.

- Ein erhöhter RA/LA-Quotient bei Patienten mit pcPH kann bei Patienten mit Linksherzerkrankungen in der kardiologischen Praxis den Anstoß geben, eine weitere invasive Abklärung zu veranlassen.

\section{Interessenkonflikt}

Matthias Held erklärt, dass er Beraterhonorare von Bayer Healthcare, Boehringer Ingelheim, Janssen, und MSD erhalten hat und Vortragshonorare von Astra Zeneca, Bayer HealthCare, Berlin Chemie, Boehringer Ingelheim, Bristol Myers Squibb, Daichi Sankyo, Janssen, MSD, Pfizer, Santis erhalten hat. 
Gülmisal Güder erklärt, dass sie Fördermittel des Bundesministeriums für Bildung und Forschung, der Deutschen Herzstiftung und der Deutschen Gesellschaft für Kardiologie erhalten und war beratend bzw. als Referentin für die Firmen AstraZeneca, Abiomed, Bayer, Boehringer Ingelheim, Novartis, Orion GmbH, Pfizer und Vifor Pharma Germany tätig. Mit der aktuellen Studie gibt es keine Interessenkonflikte. Franziska Joa erklärt, dass sie Vortragshonorare von Berlin- Chemie, Bayer, Boehringer Ingelheim, Actelion, Johnson and Johnson erhalten hat.

Elena Pfeuffer-Jovic erklärt, Reisekostenunterstützung von Actelion, Boehringer Ingelheim, Novartis und OMT erhalten zu haben. Simon Weiner, Jörg Hoffmann und Johanna Walthelm-Hösel erklären keine Interessenkonflikte zu haben.

Literatur

[1] Vachiéry JL, Tedford R, Rosenkranz S et al. Pulmonary hypertension due to left heart disease. The European respiratory journal 2019; 53 (1): 1801897 doi:10.1183/13993003.01897-2018

[2] Gerges C, Gerges M, Lang MB et al. Diastolic Pulmonary Vascular Pressure Gradient. A Predictor of Prognosis in "Out-of-Proportion" Pulmonary Hypertension. CHEST 2013; 143 (3): 758-766. doi:10.1378/chest.12-1653

[3] Rosenkranz S, Hoeper M, Huscher D et al. Targeted Therapy of Pulmonary Hypertension in Patients With Heart Failure and Preserved Ejection Fraction: Safety and Efficacy in Comparison to IPAH in COMPERA. Circulation 2015; 132: A19944

[4] Rosenkranz S, Gibbs JS, Wachter R et al. Left ventricular failure and pulmonary hypertension. Eur Heart J 2015; 37 (12): 942-954. doi:10.1093/eurheartj/ehv512

[5] Galie N, Humbert M, Vachiery JL et al. ESC/ERS Guidelines for the diagnosis and treatment of pulmonary hypertension: The Joint Task Force for the Diagnosis and Treatment of Pulmonary Hypertension of the European Society of Cardiology (ESC) and the European Respiratory Society (ERS): Endorsed by: Association for European Paediatric and Congenital Cardiology (AEPC), International Society for Heart and Lung Transplantation (ISHLT). European Heart Journal 2016; 37 (1): 67-119. doi:10.1093/ eurheartj/ehv317

[6] Magne J, Pibarot P, Sengupta PP et al. Pulmonary hypertension in valvular disease: a comprehensive review on pathophysiology to therapy from the HAVEC Group. JACC Cardiovasc Imaging 2015; 8 (1): 83-99. doi:10.1016/ j.jcmg.2014.12.003

[7] Ponikowski P, Voors A, Anker S et al. 2016 ESC Guidelines for the diagnosis and treatment of acute and chronic heart failure: The Task Force for the diagnosis and treatment of acute and chronic heart failure of the European Society of Cardiology (ESC). Developed with the special contribution of the Heart Failure Association (HFA) of the ESC. European journal of heart failure 2016; 18 (8): 891-975. doi:10.1093/eurheartj/ehw128

[8] Bonderman D, Ghio S, Felix S et al. Riociguat for patients with pulmonary hypertension caused by systolic left ventricular dysfunction: a phase IIb double-blind, randomized, placebo-controlled, dose-ranging hemodynamic study. Circulation 2013; 128 (5): 502-511. doi:10.1161/ CIRCULATIONAHA. 113.001458

[9] Hoendermis E, Liu L, Hummel Y et al. Effects of sildenafil on invasive haemodynamics and exercise capacity in heart failure patients with preserved ejection fraction and pulmonary hypertension. A randomized controlled trial. European heart journal 2015; 36 (38): 2565-2573. doi:10.1093/eurheartj/ehv336

[10] Redfield M, Chen H, Borlaug B et al. Effect of phosphodiesterase-5 inhibition on exercise capacity and clinical status in heart failure with preserved ejection fraction. A randomized clinical trial. JAMA 2013; 309 (12): 1268-1277. doi:10.1001/jama.2013.2024
[11] Guazzi M, Vicenzi M, Arena R et al. Pulmonary hypertension in heart failure with preserved ejection fraction: a target of phosphodiesterase-5 inhibition in a 1-year study. Circulation 2011; 124 (2): 164-174. doi:10.1161/CIRCULATIONAHA.110.983866

[12] Opitz CF, Hoeper MM, Gibbs JS et al. Pre-Capillary, Combined, and PostCapillary Pulmonary Hypertension: A Pathophysiological Continuum. J Am Coll Cardiol 2016; 68 (4): 368-378. doi:10.1016/j.jacc.2016.05.047

[13] Guazzi M, Naeije R. Pulmonary Hypertension in Heart Failure. Pathophysiology, Pathobiology, and Emerging Clinical Perspectives. Journal of the American College of Cardiology 2017; 69 (13): 1718-1734. doi:10.1016/j.jacc.2017.01.051

[14] EU Clinical Trials Register: EudraCTNumber 2017-003688-37.

[15] Rudski LG, Lai WW, Afilalo J et al. Guidelines for the echocardiographic assessment of the right heart in adults: a report from the American Society of Echocardiography endorsed by the European Association of Echocardiography, a registered branch of the European Society of Cardiology, and the Canadian Society of Echocardiography. J Am Soc Echocardiogr 2010; 23: 685-713. doi:10.1016/j.echo.2010.05.010

[16] Laboratories ATSCoPSfCPF. ATS statement: guidelines for the six-minute walk test. Am J Respir Crit Care Med 2002; 166: 111-117. doi:10.1164/ ajrccm.166.1.at1102

[17] American Thoracic S, American College of Chest P. ATS/ACCP Statement on cardiopulmonary exercise testing. Am J Respir Crit Care Med 2003; 167: 211-277. doi:10.1164/rccm.167.2.211

[18] Rosenkranz S, Behr ], Ewert R et al. [Right heart catheterization in pulmonary hypertension]. Dtsch Med Wochenschr 2011; 136: 2601-2616. quiz 2617-2620. doi:10.1055/s-0031-1292858

[19] Galiè N, Hoeper MM, Humbert M et al. ESC Commitee for Practice Guidelines (CPG). Guidelines for the diagnosis and treatment of pulmonary hypertension: the Task Force for the Diagnosis and Treatment of Pulmonary Hypertension of the European Society of Cardiology (ESC) and the European Respiratory Society (ERS), endorsed by the International Society of Heart and Lung Transplantation (ISHLT). Eur Heart J 2009; 30: 24932537. doi:10.1093/eurheartj/ehp297

[20] Hoeper M, Humbert M, Souza R et al. A global view of pulmonary hypertension. The Lancet Respiratory Medicine 2016; 4 (4): 306-322. doi:10.1016/S2213-2600(15)00543-3

[21] Tuder RM, Archer SL, Dorfmüller P et al. Relevant issues in the pathology and pathobiology of pulmonary hypertension. J Am Coll Cardiol 2013; 62 (Suppl. 25): D4-D12. doi:10.1016/j.jacc.2013.10.025

[22] Ghio S, Gavazzi A, Campana C et al. Independent and additive prognostic value of right ventricular systolic function and pulmonary artery pressure in patients with chronic heart failure. J Am Coll Cyrdiol 2001; 37 (1): 183-188. doi:10.1016/s0735-1097(00)01102-5

[23] Kovacs G, Berghold A, Scheidl S. Pulmonary arterial pressure during rest and exercise in healthy subjects. A systematic review. European Respiratory Journal 2009; 34 (4): 888-894. doi:10.1183/09031936.00145608

[24] Lau EM, Humbert M, Celermajer DS. Early detection of pulmonary arterial hypertension. Nat Rev Cardiol 2015; 12: 143-155. doi:10.1038/ nrcardio.2014.191

[25] Simonneau G, Montani D, Celermajer D et al. Haemodynamic definitions and updated clinical classification of pulmonary hypertension. The European Respiratory Journal 2019; 53 (1): 1801913 doi:10.1183/ 13993003.01913-2018

[26] Rosenkranz S, Diller GP, Dumitrescu D et al. Hämodynamische Definition der pulmonalen Hypertonie. Kommentar zu der vorgeschlagenen Änderung durch das $6^{\text {th }}$ World Symposium on Pulmonary Hypertension. Deutsche medizinische Wochenschrift 2019; 144 (19): 1367-1372. doi:10.1055/a-0918-3772 